\title{
Tendências e perspectivas de pesquisa sobre repositórios digitais no Brasil: uma análise de Rede Sociais (ARS)
}

\author{
Trends and perspectives of research on digital repositories in Brazil: a \\ Social Network analysis (ARS).
}

\section{Tendencias y perspectivas de investigación sobre digital repositorios en Brasil: un análisis de Red Social (ARS).}

\author{
Ivanilma de Oliveira Gama | ivanilmagama@gmail.com \\ Centro Federal de Educação Tecnológica Celso Suckow da Fonseca. Rio de Janeiro, Brasil.
}

Lidiane dos Santos Carvalho | lidiane.carvalho@unirio.br

Fundação Oswaldo Cruz, Instituto de Comunicação e Informação Científica e Tecnológica em Saúde. Rio de Janeiro, Brasil. Universidade Federal do Estado do Rio de Janeiro. Rio de Janeiro, Brasil.

\section{Resumo}

O objetivo geral desta pesquisa é mapear a rede de coautoria científica de pesquisadores brasileiros que publicaram sobre a temática dos repositórios digitais, além de, identificar os atores com maior índice de centralidade na democratização do conhecimento sobre o tema nas instituições de ensino e pesquisa no Brasil. Para a presente investigação empregou-se o Método Relacional de Análise Informacional (MRAI) que parte das seguintes dimensões: quantitativas - as técnicas bibliométricas e medidas de análise de redes sociais (ARS) e qualitativas - categorias analíticas da teoria social de Pierre Bourdieu e de seus conceitos como campo científico e capital social. Para a análise dos dados, fez-se uso do software UCINET 5.o e para os gráficos, utilizou-se também o VOSviewer. Os resultados evidenciam a maior incidência dos pesquisadores que atuam na Universidade Federal de Minas Gerais com alto índice de citações e centralidade de grau na área, seguidos dos que estão vinculados a Universidade Federal de São Carlos e a Fundação Oswaldo Cruz (Fiocruz). Por fim, recomenda-se a popularização dos repositórios institucionais nas universidades e institutos de pesquisa pela formação de elos entre atores centrais a fim de promover a democratização do acesso aberto a informação científica no Brasil.

Palavras-chave: Repositórios digitais; Análise de Redes Sociais; Informação Científica; Acesso Aberto; Evolução da Ciência; Comunicação Científica; Ciência da Informação. 


\section{Abstract}

The general objective of this research is to map the network of scientific co-authorship of Brazilian researchers publisher about the theme digital repositories, as well as to identify the actors with the highest index of centrality in the democratization of knowledge about this theme in teaching and research institutions in Brazil. For the present research, the Relational Method of Informational Analysis (RMAI) was used, starting from quantitative dimensions: bibliometric techniques and measures of analysis of social network (SNA) and qualitative aspects: analytical categories of the social theory of Pierre Bourdieu and his concepts such as scientific field and social capital. For the analysis of the data, we made use of the software UCINET 5.0 and for the graphs, the VOSviewer. The results show the incidence of researchers working at the Federal University of Minas Gerais with a high index of citations and centrality of the degree in the structure, and the other actors are the Federal University of São Carlos and the Oswaldo Cruz Foundation (FIOCRUZ). Finally, it is recommended the popularization of institutional repositories in universities and research institutes through the formation of links between central actors with the strategy to promote the democratization of open access for the scientific Information in Brazil.

Keywords: Digital repositories; Analysis of Social Networks (SNA); Scientific Information; Open Access; Evaluation of Science; Scientific Communication; Information Science.

\section{Resumen}

El objetivo general de esta investigación es mapear la red de coautoria científica de investigadores brasileños que publicaron sobre la temática de los repositorios digitales, además de identificar a los actores con mayor índice de centralidad en la democratización del conocimiento sobre el tema en las instituciones de enseñanza e investigación en Brasil . Para la presente investigación se empleó el Método Relacional de Análisis Informacional (MRAI) que parte de las siguientes dimensiones: cuantitativas - las técnicas bibliométricas y medidas de análisis de redes sociales (ARS) y cualitativas - categorías analíticas de la teoría social de Pierre Bourdieu y de sus conceptos como campo científico y capital social. Para el análisis de los datos, se utilizó el software UCINET 5.0 y para los gráficos, se utilizó también el VOSviewer. Los resultados evidencian la mayor incidencia de los investigadores que actúan en la Universidad Federal de Minas Gerais con alto índice de citas y centralidad de grado en el área, seguidos de los que están vinculados a la Universidad Federal de São Carlos y la Fundación Oswaldo Cruz (Fiocruz). Por último, se recomienda la popularización de los repositorios institucionales en las universidades e institutos de investigación por la formación de eslabones entre actores centrales a fin de promover la democratización del acceso abierto a la información científica en Brasil.

Palabras clave: Repositorios digitales; Análisis de redes sociales; Información científica; Acceso abierto; Evaluación de la ciencia; Comunicación científica; Ciencias de la Información.

\section{Introdução}

No campo da Ciência, a pesquisa é a base para a consolidação e desenvolvimento de diversas áreas. Além disso, é a relevância e reconhecimento social da produção de conhecimento científico para a resolução de problemas inerentes a condição humana, que concerne para um pesquisador, um grupo de pesquisa e instituições científicas, autoridade científica, seja em mercados nacionais e/ou internacionais.

Com efeito, a comunicação cientifica, propiciou não somente compartilhar a informação entre os pares, mas também fomentar a colaboração entre pesquisadores e grupos de pesquisa de diferentes regiões ou Países. A colaboração pode ser entendida como uma predisposição do ator social para compartilhar conhecimento, informações, recursos, direitos, deveres e responsabilidades. Esta colaboração científica entre pesquisadores e laboratórios, propicia que o conteúdo produzido em determinada pesquisa, seja comunicado com a partilha das responsabilidades e da descoberta científica.

Doponto devista do acesso aberto, os repositórios digitais representam instrumentosinfocomunicacionais fundamentais para a democratização da informação científica. Os repositórios tem sido destaque neste 
movimento no Brasil, deste o ano de 2008 com o pioneirismo do Arca $^{1}$ e sequencialmente em constante popularização nas instituições de ensino e pesquisa do País. Desse modo, como objetivo geral, esta pesquisa quer mapear a rede de coautoria científica de pesquisadores brasileiros sobre a temática repositórios digitais, e como objetivos específicos quer identificar os atores com maior índice de centralidade para fornecer apontamentos para a democratização do conhecimento sobre o tema nas instituições de ensino e pesquisa no Brasil.

Para a presente investigação empregou-se o se o Método Relacional de Análise Informacional (MRAI). Esta metodologia é particularmente interessante para os estudos de avaliação da ciência, porque congrega as seguintes dimensões: quantitativas - as técnicas bibliométricas e medidas de análise de redes sociais (ARS) ${ }^{\mathrm{i}}$ e qualitativas - categorias analíticas da teoria social de Pierre Bourdieu e de seus conceitos como campo científico e capital social. Para a análise dos dados, fez-se uso do software UCINET 5.0 e para os gráficos, utilizou-se também o VOSviewer.

Os resultados permitem apresentar a conformação de uma estrutura social de produção de conhecimento em relação à temática estudada e seus autores centrais. Evidencia-se que o processo de comunicação e cooperação científica é primordial na construção de capital social e volume de capital simbólico dos atores, especialmente no que se refere às relações de coautoria estabelecida pelos mesmos e a mobilização de recursos.

Por fim, sugere-se que os atores identificados na rede, participem na mobilização de grupos para a participação consórcios ou da fixação de convênios interinstitucionais específicos para o desenvolvimento de tecnologias da informação e da comunicação aplicado aos repositórios digitais. Especificamente em relação à rede estudada, uma abertura estrutural pode ampliar a promoção e democratização do acesso aberto na medida em que o conhecimento sobre o desenvolvimento de repositórios digitais nas instituições públicas de ensino e pesquisa em diferentes regiões do Brasil avançam.

\section{Abordagem teórica}

A abordagem teórica fundamenta-se em uma revisão dos pontos de vista históricos sobre o desenvolvimento dos repositórios digitais, da função da comunicação científica no campo científico e a da formação de redes de pesquisa. Enquanto o conceito de campo científico ${ }^{3,63,64}$ expressa o espaço da ações e trocas relacionadas a conformação das práticas cientificas, o conceito de capital social ${ }^{3,59,63,64}$ refere-se a energia mobilizada por um ou mais atores.

\subsection{Uma perspectiva histórica dos Repositórios Digitais.}

A segunda metade do século XX tornou-se uma época em que as transformações propiciadas pelas mudanças no modo de comunicação e no fluxo informacional mudaram de forma significativa os ambientes. Dentre essas, pode-se destacar o marco do surgimento da Internet, que possibilitou a transmissão informacional de modo mais amplo. Essas alterações atuaram de forma pragmática no ambiente acadêmico, permitindo a ampliação da disseminação da produção científica para todos os indivíduos. Neste cenário, ocorre a eclosão dos Repositórios Institucionais (RI), que permitiram a propagação do conteúdo digital produzido nas universidades e centros de pesquisas.

Os repositórios digitais, para Lynch ${ }^{6}$, podem ser definidos como "[...] um conjunto de serviços que a universidade oferece para os membros de sua comunidade para o gerenciamento e a disseminação de conteúdos digitais, criados pela instituição e membros da sua comunidade”. Em relação a sua organização social, a autora ${ }^{6}$ define que "É essencialmente um compromisso organizacional com a gestão, desses

i Os dados foram coletados durante a disciplina de Métodos e Técnicas de análise de Redes Sociais ministrada pela Professora Doutora Lidiane Carvalho, no âmbito do Mestrado Profissional em Biblioteconomia da Universidade Federal do Estado do Rio de Janeiro (PPGB-UNIRIO) e como objeto empírico de investigação definiu-se o tema repositórios digitais no Brasil. 
conteúdos digitais, inclusive preservação de longo prazo, quando apropriado, bem como organização e acesso ou distribuição" ${ }^{6}$.

Até o ano de 2010, o Directory of Open Access Repositories (OpenDOAR) ${ }^{\mathrm{ii}}$ registrou cerca de 1.800 repositórios vinculados a Universidades e Centros de Pesquisa em todo o mundo. Destes, existem 91 repositórios ligados a instituições brasileiras e muitas pesquisas estão se desenvolvendo sobre a temática do RI, visando compreender a configuração técnica e operacional, assim como as razões e necessidades para o seu desenvolvimento.

Desse modo, compreendem-se os "repositórios digitais" como uma ferramenta/dispositivo/artefato tecnológico com a finalidade de mediação informacional cujo objetivo é aproximar instituições e autores por meio do compartilhamento da informação científica. Gomes e Rosa ${ }^{6}$ percebem que a expansão do repositório dentro da instituição, motiva a busca de pesquisadores externos nos programas de pósgraduação e a ampliação do número de citações dos conteúdos disponíveis. Guimarães, Silva e Noronha7 afirmam que, além de seu caráter de gerenciamento e disseminação, um papel a ser considerado é o de "guia para avaliação"; assim sendo, o repositório torna-se um veículo pelo qual é possível dispor de uma estratégia de gestão institucional da pesquisa.

Tendo em vista o desenvolvimento das pesquisas e a divulgação de seus resultados, as transformações trazidas pela Web 2.0 e pelos RI confluem para o reconhecimento científico dos pesquisadores e das instituições ${ }^{4}$. O aumento da visibilidade da ciência nos ambientes sociais torna-se uma resposta aos anseios da sociedade, além de uma prestação de contas aos investimentos realizados.

\subsection{Comunicação Científica}

A comunicação científica é uma ação orientada a divulgação do conhecimento científico entre pares e seu objetivo inicial está focado segundo Meadows ${ }^{10}$ eBacon ${ }^{4,57}$ na difusão do conhecimento proveniente de pesquisas científicas, realizados por meio de uma metodologia científica sistemática e da comunicação para a ciência e da comunicação dos resultados obtidos. A comunicação científica está vinculada diretamente as políticas de desenvolvimento cientifico e tecnológico dos países. Enquanto processo, envolve o compartilhamento, os fluxos e as transferências de informação, além dos dispositivos formais e informais que são instrumentos que veiculam os achados, descobertas ou inovações provenientes de determinada pesquisa.

O conceito "comunicação científica", criado por John Bernal na década de 1940, determina um amplo processo de geração e transferência de informação científica ${ }^{7}$ e para Menzel7 $^{7}$, a comunicação científica possui sete funções, a saber:

1) fornecer respostas a perguntas específicas; 2) contribuir para a atualização profissional do cientista no campo específico de sua atuação; 3) estimular a descoberta e a compreensão de novos campos de interesse; 4) divulgar as tendências de áreas emergentes, fornecendo aos cientistas ideia da relevância de seu trabalho; 5) testar a confiabilidade de novos conhecimentos, diante da possibilidade de testemunhas e verificações; 6) redirecionar ou ampliar o rol de interesse dos cientistas; 7) fornece feedback para aperfeiçoamento da produção do cientista.

O ambiente universitário, de acordo com Meadows ${ }^{10}$ reúne as competências e conhecimentos necessários para a evolução do método e conhecimento científico. O surgimento das universidades na Idade Média,

ii The Directory of Open Acess Repositories (OpenDOAR) consiste em uma organização mantida pela SHERPA Services, o qual visa recolher e atribuir metadados que permitam a classificação e análise mais precisas para o uso dos repositórios. A OpenDOAR constrói uma listagem de todos os repositórios relacionados a Universidades e Centro de Pesquisas espalhados pelo mundo. Essa surge do trabalho in loco realizado pela equipe do Diretório ${ }^{8}$. 
segundo Le Coadic ${ }^{10,11}$, foi um fator decisivo para o surgimento da ciência organizada, ou seja, estruturada para sustentação de programas de formação e desenvolvimento da pesquisa.

Nesse contexto, os periódicos científicos exercem um papel importante no sentido de possibilitar maior comunicação no campo científico. A publicação de trabalhos nesses veículos projeta os pesquisadores a uma instância de renome, possibilitando o investimento financeiro nas pesquisas desenvolvidas por estes.

O fluxo de informação científica, segundo Garvey7, vale-se de dois canais para a comunicação de trabalhos científicos: os formais e os informais. Os formais incluem as publicações impressas; e os informais se designam pela oralidade. Ambos apresentam diferenciação em sua estrutura, contudo cabe salientar suas relevâncias no processo comunicacional e se complementam. Ou seja, as permutas informacionais traçadas na informalidade entre os pesquisadores, permitem que se desenvolvam pesquisas, passando para o campo da formalidade científica. A formalidade científica produz alguns objetos, quais sejam, teses, dissertações, artigos científicos e pré-prints, entre outros. Nessa conjuntura, as metodologias de avaliação da ciência utilizam-se de métodos e técnicas, composto por medidas e ferramentas, para evidenciar as práticas científicas.

Como disciplina, a comunicação cientifica para configurar-se como tal, reúne métodos técnicos e abordagens teórico empíricas capazes de articular o entendimento do mundo cientifico na perspectiva do participante e do observador. A princípio, os repositórios configuram-se como um canal de mediação para acesso e uso da informação científica formal. Neste contexto, é função da comunicação na ciência, a criação de dispositivos de interação e linguagens de aproximação entre o especialista e o cidadão.

\section{Procedimentos metodológicos}

Os dados que conformaram o campo empírico de análise para a evidenciação das redes de coautoria foram identificados a partir da produção científica dos pesquisadores do campo da Ciência da Informação que tiveram os seus artigos publicados e indexados na base de dados na base Web of Science ${ }^{31}$ até novembro de 2016. Como objetivos específicos, exploraram-se quais seriam os atores com maior índice de centralidade no campo dos repositórios no cenário nacional e a relevância dos relacionamentos nesse contexto. Para tal fim, adotou-se a metodologia de Análise de Rede Sociais (ARS) e percorreram-se os dados bibliométricos recolhidos. Para recolher tais informações, foi utilizado o seguinte procedimento: Tópico: (Repository). Após serem obtidos mais de três mil documentos, decidiu-se realizar um refinamento a partir da delimitação do país (Brasil) e por categorias da Web of Science ${ }^{31}$ (Information Science Library), além de um recorte temporal, considerando artigos de todos os anos desde 1945 até 2016.

A opção pela Web of Science ${ }^{31}$ ocorre por conta da amplitude na indexação de periódicos no campo da CI. Por conta desta amplitude mundial na indexação da literatura científica, atualmente, a base abrange cerca de 12.00o periódicos, além do conteúdo de cinco coleções: Science Citation Indez Expanded (SCI-EXPANDED), Social Sciences Citation Index (SSCI), Arts \& Humanities Citation Index (A\&HCI), Conference Proceedings Citation Index-Science (CPCI-S) e Conference Proceeding Citation Index - Social Science \& Humanities (CPCI-SSH).

Para a obtenção dos dados bibliométricos, assim como os grafos de redes apresentados neste estudo, utilizouse o software UCINET 5.o, a fim de calcular a "centrality degree", que pode ser definida como o número de laços que incidem sobre um nó ${ }^{12}$ e representa o volume de capital social mobilizado na estrutura científica.

Em contrapartida, a Web of Science ${ }^{31}$ apresenta ferramentas para análise de citações, referências, índice $h$ para inquirir os autores de maior relevância na área. Exploraram-se os dados bibliométricos referentes ao índice $h$ fornecidos pela Web of Science ${ }^{31}$ também para quantificar a atividade científica e mensurar o impacto de pesquisadores, baseando-se nos seus artigos com maior índice de citação. Os resultados estatísticos encontrados se deram com o cálculo do maior número de artigos de um autor específico que tivesse, ao menos, o mesmo número de citações². 
Os recursos metodológicos acima apresentados compõem o Método Relacional de Análise de Redes Sociais (MRAI). A MRAI é oriunda da abordagem sociocultural da informação que reconhece a "informação construída" pelos sujeitos que procuram compartilhar experiências em um processo infocomunicacional de significação coletiva ${ }^{9,58-65}$

Quadro 2: Centralidade de grau e Índice de citações

\begin{tabular}{|l|l|}
\hline $\begin{array}{l}\text { Medidas de ARS } \\
\text { Centrality Degree and Betweenness }\end{array}$ & $\begin{array}{l}\text { Índice de Citação } \\
\text { (ISI, Google Scholar, Citeseer X, etc.). }\end{array}$ \\
\hline CONTEXTO/ESTRUTURA & CONTEÚDO \\
\hline Freeman ${ }^{13,66}$ baseado na teoria dos grafos. & $\begin{array}{l}\text { Garfield } \\
\text { ciên,68 funda o ISI em } 1958 \text { para avaliação da }\end{array}$ \\
\hline $\begin{array}{l}\text { Considera a distância entre nós (atores) e } \\
\text { capacidade de mobilização até outro ponto } \\
\text { da mesma estrutura. }\end{array}$ & $\begin{array}{l}\text { Avalia a relevância do conteúdo para a área com base } \\
\text { no número de menções por outros atores. }\end{array}$ \\
\hline
\end{tabular}

Fonte: Carvalho (2017). ${ }^{59}$

Por último, fazem-se necessárias algumas orientações para a melhor compreensão dos dados: a nomenclatura utilizada para cada ator da rede foi representada conforme disponível na base de dados. Assim, possibilitou a busca posterior dos dados e sua identificação na Web of Science ${ }^{31}$.

\section{Apresentação dos resultados}

O estudo realizado por meio dos dados coletados na base Web of Science ${ }^{31}$ permitiu que fosse emitida

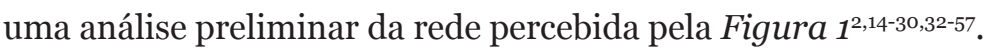

Figura 1: Rede de Coautoria de Repositórios no Brasil (2016)

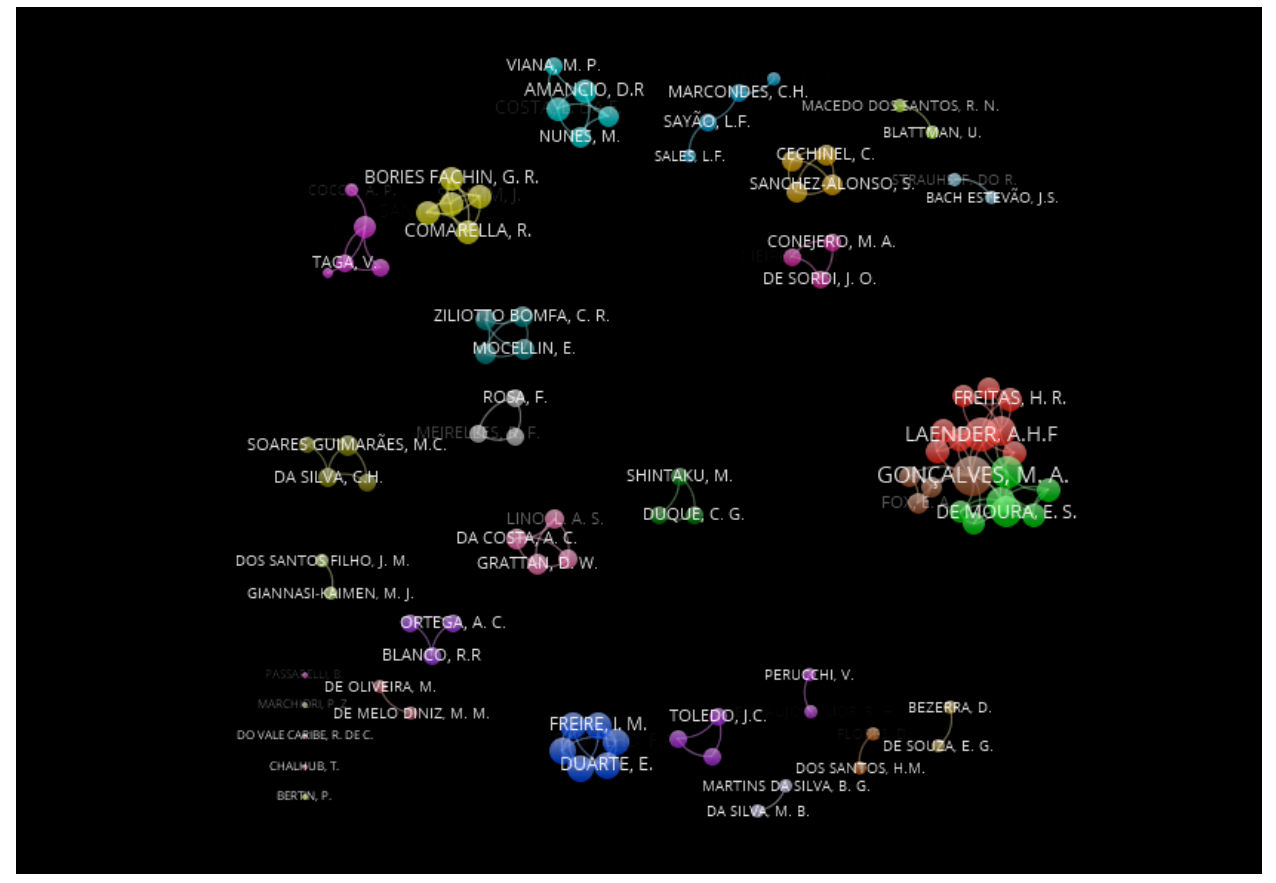

Fonte: As autoras (2016) 
A Figura 1 mostra um panorama geral da rede de coautorias existente sobre o assunto repositório no universo da Ciência da Informação; esse quadro pode ser visualizado pela Web of Science ${ }^{31}$. Nesse sentido, a base de dados, em sua análise de resultados das informações recuperadas, forneceu os 13 autores que mais se destacam nesse cenário, conforme Tabela 1.

Tabela 1: Índice de Centralidade (Web of Science)

\begin{tabular}{|c|c|}
\hline Autores & Índice de Citação (\%) \\
\hline \multicolumn{2}{|l|}{ Web of Science } \\
\hline GONÇALVES, MA ${ }^{14,18,20,27}$ & 9,756 \\
\hline GUIMARÃES, MCS ${ }^{15,16,17}$ & 7,317 \\
\hline LAENDER, AHF $18,19,20$ & 7,317 \\
\hline RODRIGUES, RS $21,22,23$ & 7,317 \\
\hline AMANCIO, DR ${ }^{24,25}$ & 4,878 \\
\hline COSTA, LF 24,25 & 4,878 \\
\hline DA SILVA, AS 26,27 & 4,878 \\
\hline DE MOURA, ES 26,27 & 4,878 \\
\hline MARCONDES, $\mathrm{CH}^{28,29}$ & 4,878 \\
\hline RIBEIRO-NETO, $\mathrm{B}^{19,20}$ & 4,878 \\
\hline RODRIGUES, JG ${ }^{15,16}$ & 4,878 \\
\hline SAYÃO, LF 29,30 & 4,878 \\
\hline ZIVIANI, N20,26 & 4,878 \\
\hline
\end{tabular}

Fonte: Web of Science $(2016)^{31}$

As citações se configuram como meios que autenticam o desenvolvimento das ciências. Por intermédio delas, é possível mensurar as contribuições dos pesquisadores entre seus pares². Além disso, permitem uma observação sistêmica do "ambiente teórico" no qual se está inserido, como também os "circuitos acadêmicos" que lhes confere legitimidade ${ }^{2}$. Spinak ${ }^{2}$ sinaliza alguns indicadores que têm como base a citação, entre eles: "índice de atividade, afinidade, atração, imediatez, 'popularidade', isolamento, abertura e, finalmente, o de impacto".

O índice de impacto, ou também índice $h$, objetiva quantificar os trabalhos científicos de cada pesquisador e medir o impacto dos mesmos por meio dos seus papers mais citados. Lima, Strini Velho e Faria ${ }^{2}$ explicam que o valor da variável h se dá pelo cálculo do "maior número de artigos de um autor específico que possui, pelo menos, o mesmo número de citações". Na ARS, Carvalho ${ }^{66}$ explica que "a centralidade de grau, de modo complementar, nos informa sobre a capacidade de mobilização de um determinado pesquisador e do acesso aos recursos de pesquisa, enquanto a citação trata da relevância do conteúdo para a construção de novos conhecimentos".

Cabe salientar que o índice $h$ não decresce, ou seja, o autor pode estacionar no percentual de contribuição na sua área, mas não diminui o impacto das suas contribuições anteriores. Entretanto, seu aumento também não é linear, pois o fator não está ligado somente à incidência de trabalhos publicados, mas ao de citação'. $\mathrm{O}$ índice $h$ se caracteriza por indicar a produtividade do pesquisador por meio da citação, utiliza os dados disponíveis em base de dados confiáveis (Web of Science e Scopus) e não é mutável por fatores extremos.

Desse modo, os dados trazidos da Web of Science ${ }^{31}$ trazem um panorama dos autores que exercem um alto índice de citações entre seus pares. Isso lhes configura um grau de relevância entre os pesquisadores da Ciência da Informação que estudam a temática dos repositórios. Pela ótica da Ciência da Informação, os aspectos trazidos permitem uma visão dos impactos dos conceitos deixados por esses teóricos no seu ambiente. Contudo, podem-se olhar essas informações por uma perspectiva da Sociologia da Ciência e, neste 
sentido, observa-se a transformação social que a citação traz para a comunidade em que se está inserida, no caso deste artigo, investigaram-se os reflexos entre os pesquisadores que se dedicam aos repositórios na Ciência da Informação. A Tabela 2 traz informações acerca dessa visão por meio de índice de centralidade, trazido por Freeman ${ }^{12,13}$.

Os autores que se destacam pelo alto índice $h$ são prof. Dr. Marcos André Gonçalves, da Universidade Federal de Minas Gerais (UFMG); prof. Dra. Maria Cristina Soares Guimarães, da Fundação Oswaldo Cruz (Fiocruz), prof. Dr. Alberto Henrique Frade Laender, da Universidade Federal de Minas Gerais (UFMG), Prof. Dr. Diego Raphael Amâncio, da Universidade Federal de São Carlos (UFSCar), Prof. Dr. Luciano da Fontura Costa, da Universidade Federal de São Carlos (UFSCar), Prof. Dr. Altigran Soares da Silva, do Conselho Nacional de Desenvolvimento Científico e Tecnológico (CNPq), prof. Dr. Edleno Silva de Moura, da Universidade Federal do Amazonas (UFAM), prof. Dr. Carlos Henrique Marcondes de Almeida, da Universidade Federal Fluminense (UFF), prof. Dr. Berthier Ribeiro de Araujo Neto, da Universidade Federal de Minas Gerais (UFMG), prof. Dra. Jeorgina Gentil Rodrigues, Fundação Oswaldo Cruz (Fiocruz), prof. Dr. Luís Fernando Sayão, Comissão Nacional de Energia Nuclear (CNEN), prof. Dr. Nívio Ziviani, da Universidade Federal de Minas Gerais (UFMG).

Tabela 2: Índice de Centralidade (Rede de Coautoria)

\section{Autores}

\section{Grau de Centralidade de Grau (UCINET)}

\section{Rede de Coautoria}

\begin{tabular}{|c|c|}
\hline GONÇALVES, MA ${ }^{14,18,20,27}$ & 14 \\
\hline LAENDER, AHF $18,19,20$ & 10 \\
\hline ZIVIANI, N20,26 & 8 \\
\hline DE MOURA, ES 26,27 & 7 \\
\hline RIBEIRO-NETO, $\mathrm{B}^{19,20}$ & 7 \\
\hline FREIRE, IM ${ }^{32}$ & 5 \\
\hline DE AZEVEDO NETTO, C ${ }^{32}$ & 5 \\
\hline DUARTE, $E^{2,32}$ & 5 \\
\hline RAMALHO, FA ${ }^{32}$ & 5 \\
\hline DIAS, $\mathrm{GA}^{32}$ & 5 \\
\hline DE ARAUJO FREIRE, $\mathrm{GH}^{32}$ & 5 \\
\hline AMANCIO, DR ${ }^{24,25}$ & 4 \\
\hline BORGES, EN ${ }^{18}$ & 4 \\
\hline
\end{tabular}

Fonte: As autoras

Esse estudo se debruçou sobre a estrutura social pertinente ao universo empírico, o qual foi averiguado por intermédio das relações presentes nesse meio. O intuito não foi analisar somente os predicados individuais, mas perceber como isso modifica a rede e como são as interações dentro da mesma.

Por meio do UCINET 5.0, exploraram-se dois conceitos de medidas que poderiam auxiliar no objetivo desse estudo: cliques e centralidade. Os cliques, de acordo com Marteleto9, podem ser definidos como "[...] o grupo de atores no qual cada um está direta e fortemente ligado a todos os outros". Nessa conjuntura, foram identificados 22 cliques, com pelo menos três autores, que é a menor estrutura fornecida pelo software, como mostra a Figura 2. 
Figura 2: Cliques na Rede de Coautoria

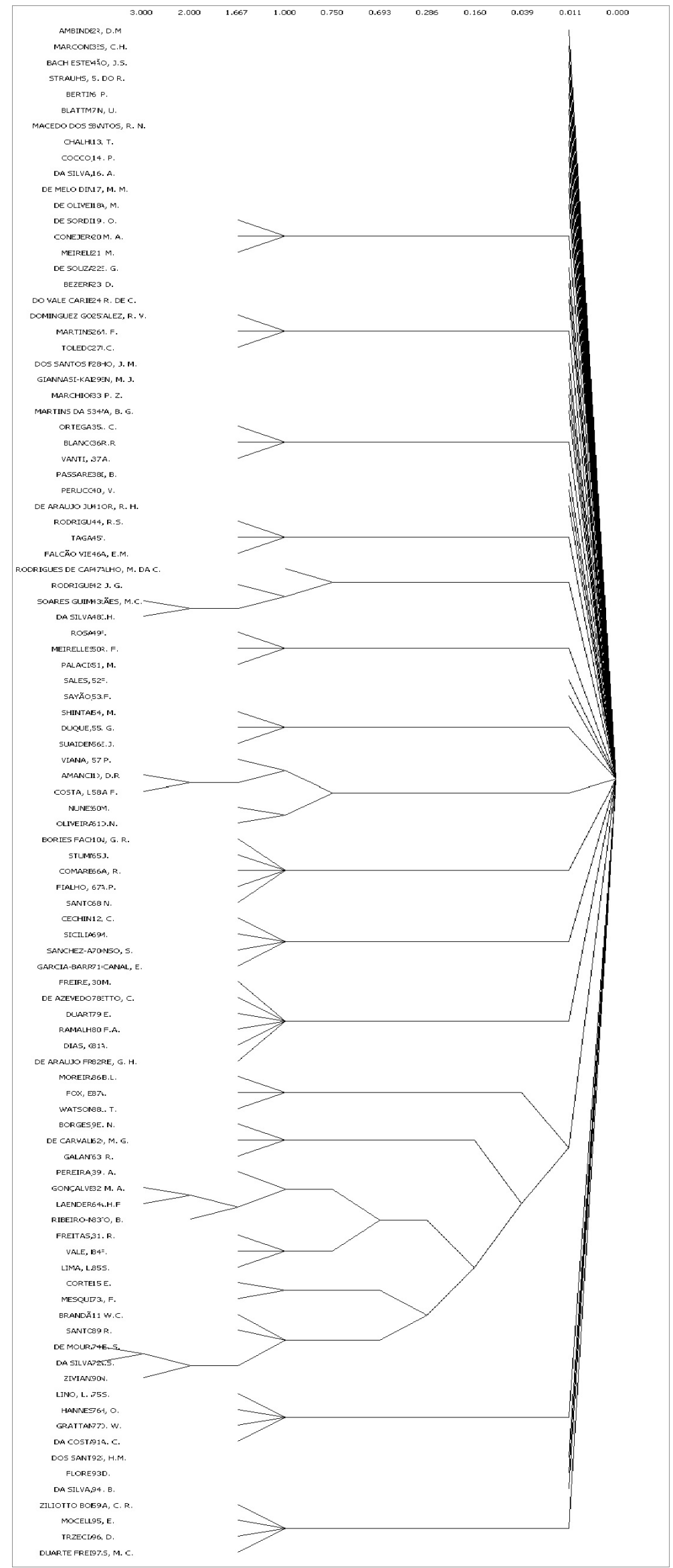

Fonte: As autoras (2016). 
Pelos dados analisados, é possível perceber que os atores que mais se destacam participam igualmente do maior número de cliques. Como, por exemplo, Gonçalves, MA ${ }^{14,18,20,27}$, que tem a incidência em cinco cliques. Isso vai ao encontro de conceito de "volume de capital social" de um ator social, trazido por Bourdieu exprime a extensão das redes de relações que o mesmo possui ou consegue impulsionar. Ligado a isso, é possível verificar que os atores que possuem mais "volume do capital simbólico" perante a rede, conseguem realizar um maior número de ligações. Percebe-se um ciclo dentro da rede em relação aos autores com maior número de coautoria; quanto maior o volume do capital simbólico desses, maior será o volume de capital social, o que confere a eles o título de agentes mobilizadores.

Partindo para o outro ponto de medida, a centralidade, definido por Freeman ${ }^{12,13}$ como a soma das distâncias de um nó para o outro, a distância é definida como comprimento, em links, do mais curto caminho. Em relação ao fluxo informacional, essa medida ajuda a identificar índice de temporalidade dos dados na rede. A centralidade permite saber o posicionamento do ator em relação às trocas e à comunicação na rede ${ }^{33}$. Desse modo, percebe-se que quanto mais central o indivíduo na rede, maior o seu poder dentro dela.

Uma comparação entre a Tabela 1, em que se utilizou o índice $h$, e a Tabela 2, em que foram usadas informações do software UCINET, baseados nos cálculos de Freeman ${ }^{12,13}$, concede a noção de que o fato de o autor ser mais citado não implica necessariamente a ideia de ele ter mais impacto na rede. Isso pode ser explicado pelo conceito de "aberturas estruturais", ou seja, um ator que se intercambiou entre a rede em diversas relações, maximiza os seus contatos, o que emite a ele uma centralidade maior do que os demais, como averiguado, por exemplo, no caso do autor Ziviani ${ }^{20,26}$. Na Tabela 1, ele é visto com um de menor índice de citações na área, entretanto, na análise de redes sociais, percebe-se que ele se sobressai aos demais, se tornando o terceiro com maior índice de centralidade. O inverso também ocorre, como, por exemplo, Sayão, L. F29,30 encontra-se como um dos mais citados na Web of Science ${ }^{31}$, contudo, sua relação na rede social é baixa e ele não se encontra entre os 13 primeiros.

Os autores que se destacam pelo alto índice de centralidade são prof. Dr. Marcos André Gonçalves, da Universidade Federal de Minas Gerais (UFMG); prof. Dr. Alberto Henrique Frade Laender, da Universidade Federal de Minas Gerais (UFMG), prof. Dr. Nivio Ziviani, da Universidade Federal de Minas Gerais (UFMG), prof. Dr. Edleno Silva de Moura, da Universidade Federal do Amazonas (UFAM), Prof. Dr. Berthier Ribeiro de Araujo Neto, da Universidade Federal de Minas Gerais (UFMG), prof. Dra. Isa Maria Freire, da Universidade Federal da Paraíba (UFPB) e Universidade Federal da Bahia (UFBA), prof. Dr. Carlos Xavier de Azevedo Netto, da Universidade Federal da Paraíba (UFPB), prof. Dra. Emeide Nóbrega Duarte, da Universidade Federal da Paraíba (UFPB), prof. Dra. Francisca Arruda Ramalho, da Universidade Federal da Paraíba (UFPB), prof. Dr. Guilherme Ataíde Dias, da Universidade Federal da Paraíba (UFPB), prof. Dr. Gustavo Henrique de Araújo Freire, da Universidade Federal da Paraíba (UFPB), Prof. Dr. Diego Raphael Amancio, da Universidade Federal de São Carlos (UFSCar), prof. Dr. Eduardo Nunes Borges, da Universidade Federal do Rio Grande (FURG).

Os pesquisadores da Universidade Federal de Minas Gerais apresentam alto índice de citações na área. Seguidos dos que estão vinculados a Universidade Federal de São Carlos e a Fundação Oswaldo Cruz (Fiocruz). Ao analisar os pesquisadores com alto índice de centralidade na rede são aos vinculados a Universidade Federal da Paraíba e alguns deles não possuem o alto índice de citação, mas se constituem como agentes mobilizadores.

Além disso, salienta-se que as formações de grande parte dos pesquisadores que alto índice $h$ estão ligadas à área das Ciências Exatas, tais como Física, Engenharia e Computação. Enquanto que aos que possuem alto índice de centralidade são os que têm formação na área de Biblioteconomia, o que confere uma multidisciplinaridade do campo e como isso maximiza a produção na área. Destacam-se, nesse universo, cinco autores que se encontram à parte na rede. Isso mostra que os mesmos, por não terem uma relação construída entre seus pares, se mantêm à margem da rede, o que também pode ser visto pela tabela 1. 


\section{Considerações finais}

Esta investigação, de caráter interdisciplinar, expressa o conhecimento produzido no campo dos estudos de informação sobre a temática de repositórios digitais. Por meio da análise de redes sociais (ARS) associada à observação dos dados de cliques e centralidade em comparação ao índice $h$ trazidos da análise dos resultados coletados na Web of Science identificou e descreveu: os atores que produziram conhecimento sobre o tema nos últimos anos, suas redes de pertencimento e sua capacidade de mobilização e articulação em arranjos de pesquisa em torno do tema.

Do ponto de vista da avaliação da ciência, o MRAI, como método permitiu: a) identificar os atores participantes no protagonismo sobre a implementação de repositórios no Brasil pela literatura por eles publicada devidamente indexada pela Web of Science; b) expressar quem são estes atores; c) propor a abertura estrutural da rede para promoção do acesso a informação científica.

Os indivíduos com maior centralidade de grau, na rede de coautoria estudada, apresentaram algumas peculiaridades: nem todos aqueles que reúnem altos índices de citações, se sobressaem na análise das relações de coautoria. Cabe considerar que a rede de coautoria é relação tipicamente classificada como rede de colaboração. Isso mostra que o capital social emitido nas relações sociais tange as relações comunicacionais estabelecidas por diferentes arranjos produtivos na ciência. O processo de comunicação e participação social, as trocas e compartilhamento informacional entre os atores é fundamental não só para a produção em coautoria, mas para o acesso a recursos de pesquisa e inclui desde o compartilhamento de tecnologias, experiências, metodologias até o fortalecimento do pesquisador na estrutura social.

A autoria é uma expressão simbólica da capacidade de mobilização e articulação de pesquisa de um autor, por sua vez, o acesso a capacidade de mobilização pode ser entendido na perspectiva do poder, ou do capital social - força de mobilização social. Um pesquisador ao compartilhar uma autoria, se une a outro formando elos, que expressa a rede de colaboração formada por um grupo ou diversos grupos de pesquisa em diferentes contextos. Dependendo da força de conexão de um determinado autor (centralidade de grau) com outros autores em uma rede de pesquisa, maior será o acesso aos recursos que ele pode mobilizar. Por fim, recomenda-se a popularização dos repositórios institucionais em universidades e institutos de pesquisa pela formação de elos entre atores centrais em cada clique, a fim de promover a democratização do acesso aberto a informação científica no Brasil.

\section{Referências}

1. Carvalho MCR, Silva CH, Guimarães MCS. Repositório institucional da saúde: a experiência da Fundação Oswaldo Cruz. Informação \& Sociedade: Estudos 2012, 22(1): 97-103.

2. Lima RA, Velho LMLS, Faria LIL. Bibliometria e "avaliação" da atividade científica: um estudo sobre o índice h. Perspectivas em Ciência da Informação 2012; 17(3): 3-17.

3. Bourdieu P. Escritos de educação. 9. ed. Petropólis, RJ: Vozes; 2007.

4. Lima CM; Carvalho LS. Discurso, análise de redes e avaliação dos processos de inovação. DataGrammaZero: Revista da Informação 2011 Dez; 12(6): 1-11.

5. Gomes MJ; Rosa F. Repositórios institucionais: democratizando o acesso ao conhecimento. Salvador: UFBA; 2010.

6. Rosa F, Meirelles RF, Palacios M. Institutional repository of the Federal University of Bahia: implantation and follow-up. Informação \& Sociedade: Estudos 2011; 21(1): 129-41.

7. Guimarães MCS, Silva, CH, Noronha,IH. RI é a resposta, mas qual é a pergunta? Primeiras anotações para a implementação de repositório institucional. In: Sayão LF, Toutain LMBB, Rosa FGMG, Marcondes $\mathrm{CH}$ (Orgs.). Implantação e gestão de repositórios institucionais: políticas, memória, livre acesso e preservação. Salvador: EDUFBA; 2009. p. 261-281. 
8. The directory of open acess repositories [homepage na internet] [acesso em 11 dez 2016]. Disponível em: http://www.opendoar.org/

9. Marteleto RM. Análise de redes sociais - aplicação nos estudos de transferência da informação. Ciência da Informação 2001; 30(1): 71-81.

10. Meadows AJ. A comunicação científica. Brasília: Briquet Lemos; 1999.

11. Le Coadic Y. A ciência da informação. Brasilia: Briquet Lemos; 1994.

12. Borgatti SP. Centrality and network flow. Social Networks 2005; 27(1): 55-71, 2005.

13. Freeman LC. Centrality in social networks: conceptual clarification. Social Networks 1978/79; n.1: 215239.

14. Gonçalves MA, Moreira B, Fox E, Watson L. "What is a good digital library?" A quality model for digital libraries. Information Processing \& Management 2007 Set; 43(5): 1416-37.

15. Rodrigues JG, Soares Guimarães MC. Metadata standard for rare works on the web: an exploratory study in the Oswaldo Cruz Foundation. Informação \& Sociedade: Estudos 2014; 24(2): 99-110.

16. Rodrigues JG, Soares Guimaraes MC. Notes about the feminine participation in the field of health as from the collection of rare works the Manguinhos Library at the Oswaldo Cruz Foundation. Perspectivas em Ciência da Informação 2016 Mar; 21(1):119-33.

17. Rodrigues de Carvalho MC, Da Silva CH, Soares Guimaraes MC. Institutional repository in The Health Sector: the experience of the Fundação Oswaldo Cruz. Informação \& Sociedade: Estudos 2012; 22(1): 97-103.

18. Borges EN, De Carvalho M, Galante R, Gonçalves M, Laender A. An unsupervised heuristic-based approach for bibliographic metadata deduplication. Information Processing \& Management 2011 Set; 47(5): 706-18.

19. Freitas HR, Ribeiro-Neto B, Vale R, Laender A, Lima L. Categorization-driven cross-language retrieval of medical information. Journal of the American Society for Information Science and Technology 2006 Fev; 57(4): 501-10.

20. Pereira DA, Ribeiro-Neto B, Ziviani N, Laender A, Gonçalves M. A Generic Web-Based Entity Resolution Framework. Journal of the American Society for Information Science and Technology 2011 Maio; 62(5): 919-32.

21. Cocco AP; Rodrigues RS. Open Access Institutional Repositories: scenario on ibero-american countries. Informação \& Sociedade: Estudos 2014; 24(2): 111-20.

22. Da Silva RA, Rodrigues RS. Open educational repository features for Brazilian sign language users. Transinformação 2013; 25(1):65-79.

23. Rodrigues RS, Taga V, Falcão Vieira, EM. Open educational resources for Brazilian system of open university: preliminary studies. Perspectivas em Ciência da Informação 2011 Set; 16(3): 181-207.

24. Amancio DR, Nunes M, Oliveira O, Costa L. Using complex networks concepts to assess approaches for citations in scientific papers. Scientometrics 2012 Jun; 91(3):827-42.

25. Viana MP, Amancio DR, Costa LF. On time-varying collaboration networks. Journal of Informetrics 2013; 7(2): 371-78.

26. Brandão WC, Santos R, Ziviani N, De Moura E, Da Silva A. Learning to expand queries using entities. Journal of the Association for Information Science and Technology 2014 Set; 65(9): 1870-83.

27. Cortez E, Da Silva A, Gonçalves M, Mesquita F, De Moura E. A flexible approach for extracting metadata from bibliographic citations. Journal of the American Society for Information Science and Technology, 2009 Jun.; 60(6): 1144-58.

28. Ambinder DM, Marcondes, $\mathrm{CH}$. New experiences for presenting, accessing, and reading digital scientific articles on the web. Transinformação 2013; 25(3):195-201.

29. Sayão $\mathrm{LF}$, Marcondes $\mathrm{CH}$. The challenge of the interoperability and the new perspectives for digital libraries. Transinformação 2008; 20 (2): 133-48.

30. Sales LF, Sayão LF. Cyberinfrastructure information for research: a proposal for architecture for integrating repositories and CRIS systems. Informação \& Sociedade: Estudos 2015 Dez; 25(3): 163-84. 
31. Web of Science [base de dados]. [Acesso em 11 dez 2016]. Disponível em: http://appswebofknowledge.ez39.periodicos.capes.gov.br/WOS GeneralSearch input.do?product=WOS\&search mo de=GeneralSearch\&SID=Q2MiWdcz3Xz6fJpkAyP\&preferencesSaved=.

32. Freire IM, De Azevedo Netto C, Duarte E, Ramalho F, Dias G, De Araujo Freire G. Social responsibility of information science: report on the organization of the $X$ National Meeting of Research in Information Science. Informação \& Sociedade: Estudos 2010; 20(1): 83-91.

33. Marteleto RM; Tomaél MI. Redes sociais : posições dos atores no fluxo da informação. Encontros Bibli: Revista Eletrônica de Biblioteconomia e Ciência da Informação 2006; n. esp.: 75-91.

34. Bach Estevão JS, Strauhs, FR. Information retrieval in institutional repositories: Proposal of an ontology for Historical Organizational Memory. Qualitative \& Quantitative Methods in Libraries 2015 Dez; p. 983-92.

35. Bertin P. A three-phase model proposal for the evolution of scientific communication: From first, print periodicals to current electronic communication system. Transinformação 2008; 20(1):17-28.

36. Blattmann U, Macedo dos Santos RN. Brazilian journals and that visibility in open access. Informação \& Sociedade: Estudos 2014; 24(3): 99-106.

37. Brambilla SDS. Produção científica da UFRGS representada na Web of Science (2000- ) Perspectivas em Ciência da Informação 2012;17(2): 34-50, 2012.

38. Bories Fachin GR, Stumm J, Comarella R, Fialho F, Santos N. Management of the kwonledge and vision cognitive of the institucional repositories. Perspectivas em Ciência da Informação 2009 Ago; 14(2): 220-35.

39. Cechinel C, Sicilia M, Sanchez-Alonso S, Garcia-Barriocanal E. Evaluating collaborative filtering recommendations inside large learning object repositories. Information Processing \& Management 2013 Jan; 49(1): 34-50.

40. Chalhub T. Actions for Implementation of institutional repositories in public university in Rio de Janeiro state. Informação \& Sociedade: Estudos 2012; 22(2):115-26.

41. Da Costa ACA, Lino L, Hannesch O, Grattan D. Practical applications of Sebera's Isoperms for estimating the impact on permanence of the transfer of important archival documents to more suitable storage conditions. Restaurator: International Journal for the Preservation of Library and Archival Material 2012 Maio; 33(2): 156-78.

42. De Melo Diniz MM, De Oliveira M. Thecnical production in development agencies: case study. Informação \& Sociedade: Estudos 2015 Abr; 25(1): 123-35.

43. De Sordi JO, Conejero MA; Meireles M. Bibliometric indicators in the context of regional repositories: proposing the D-index. Scientometrics $2016 \mathrm{Abr} ;$ 107(1): 235-58.

44. De Sordi JO, Meireles MA. Extraction of larger value of collaborative information systems: importance of the semantic domain of the communication protocols by the actors. Perspectivas em Ciência da Informação, 2010 Abr; 15(1): 198-19.

45. De Souza EG, Bezerra DA. Functional Requirements for Bibliographic Records in the context of the Semantic Web: The contribution of Paul Otlet. Transinformação 2016 Ago; 28(2): 143-57.

46. Do Vale Caribe RC. Application of the collection development and management in the construction of institutional repositories. Informação \& Sociedade: Estudos 2008; 18(2): 25-40.

47. Dominguez Gonzalez RV, Martins MF, Toledo JC. Managing knowledge in a service provider: a network structure-based model. Journal of Knowledge Management 2014; 18(3): 611-30.

48. Dos Santos HM, Flores D. Trusted digital repositories for digital archival documents: considerations on the preservation in long-term. Perspectivas em Ciência da Informação 2015 Jun; 20(2): 198-218.

49. Dos Santos Filho JM, Giannasi-Kaimen MJ. Digital library as an information resource for distance learning: an analysis of higher education institutions accredited for distance learning programs in the southern region of the country. Informação \& Sociedade:Estudos 2009; 19(3): 87-7.

50. Marchiori PZ. Digital libraries and learning objects repositories. Informação \& Sociedade: Estudos 2012; 22(2): 13-21.

51. Martins da Silva BG, Da Silva MB. Analysis of scientific production in information technology: A panoramic study of articles published by librarianship professors from the UnB. Biblios: Revista de Bibliotecologia y Ciencias de la Informacion 2015; 59: 18-33. 
52. Ortega AC, Blanco RR, Vanti AA. Information management in global environments: Swarm Intelligence in multilingual economic document repositories. Informação \& Sociedade: Estudos 2013; 23(1): 27-38.

53. Passarelli B. Students' collective knowledge construction in the virtual learning environment "ToLigado your school interactive newspaper". Information Research: an International Electronic Journal 2008 Mar; 13(1): 335

54. Perucchi V, De Araujo Junior RH. Scientific production about competitive intelligence of the School of Science Information of Brasilia University. Perspectivas em Ciência da Informação 2012 Jun.; 17(2): 37-56.

55. Shintaku M; Duque CG, Suaiden EJ. Federation of repositories: concepts, policies, characteristics and trends. Perspectivas em Ciência da Informação 2015 Set; 20(3): 51-66.

56. Ziliotto Bomfá CR, Mocellin E, Trzeciak D, Duarte Freitas M. Open access to digital scientific information: difficulties and tendencies. Transfinformação 2008; 20(3): 309-18.

57. Bacon F. The praise of knowledge. [acesso em 01 nov 2016]. Disponível em: https://books.google.com.

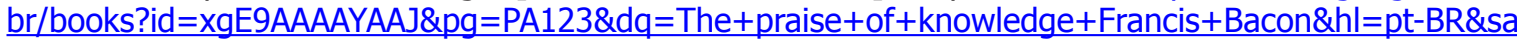
=X\&ved=0ahUKEwiEotWYh6LVAhWIh5AKHebyDIYQ6AEIIjAA\#v=onepage\&q=The\%20praise\%20of\%20 knowledge\%20Francis\%20Bacon\&f=false.

58. Carvalho LS. A abordagem sociocultural da produção de conhecimento científico. In: Marteleto RM, Pimenta RM. (org). Pierre Bourdieu e a produção social da cultura, do conhecimento e da informação. Rio de Janeiro: Garamond; 2017a. p. 190-290.

59. Carvalho LS. Integridade científica e os aspectos bioéticos do compartilhamento da informação em biobancos: uma perspectiva relacional. In: Cassimiro MC, Diós-Borges MMP (orgs). Integridade Científica, Saúde Pública, Bioética e Educação em Saúde no Instituto Oswaldo Cruz. Porto Alegre: Editora F; 2017b. p. 99-122.

60. Hjørland B. Towards a theory of aboutness, subject, topicality, theme, domain, field, content and relevance. Journal of the American Society for Information Science and Technology 2001; 52(9), p. 774 -778 .

61. Hjørland B. Domain analysis in information science: eleven approaches -traditional as well as innovative. Journal of Documentation 2002; 58, p. 422-462.

62. Marteleto RM. Conhecimento e conhecedores: apontamentos sobre a ciência, os pesquisadores e seu papel social. In: Marteleto RM, Stotz EN (org.). Conhecimento e redes sociais: diálogos de conhecimentos nas comunidades da Maré. Rio de Janeiro: Editora Fiocruz; Belo Horizonte: Editora UFMG, 2009a, p.43-62.

63. Marteleto RM, Carvalho LS. Health as a Knowledge Domain and Social Field. Journal of Documentation 2015, 42, p. 581.

64. Carvalho LS. Informação e Genética Humana: o sequenciamento de uma cultura científica. [Tese] Rio de Janeiro: Universidade Federal do Rio de Janeiro - Escola de Comunicação; 2014.

65. Carvalho LS. The Knowledge Organization (KO) Studies in the Health Field: a relacional perspective. In: $14^{\text {th }}$ International ISKO Conference, 2016; Rio de Janeiro. Rio de Janeiro: ISKO Conference; 2016.

66. Freeman LC. Centrality in social Networks conceptual clarification. Social Networks 2005, 1, p. $215-239$.

67. Garfield E. Citation indexes: new paths to scientific knowledge. Chemical Bulletin 1956, 43 (4), p. 11.

68. Garfield E. What is a significant journal? Current Contents 1970, 18, p. 5-6. 